\title{
Efficacy of pedicle screw fixation in the treatment of spinal instability and failed back surgery: a 5-year review
}

\author{
Roberto Masferrer, M.D., Carlos H. Gomez, M.D., Dean G. Karahalios, M.D., and Volker K. H. \\ Sonntag, M.D.
}

Division of Neurological Surgery, Barrow Neurological Institute, Mercy Healthcare Arizona, Phoenix, Arizona

Object. The goal of this study was to review retrospectively the outcome of 95 patients with various disorders leading to instability of the thoracolumbar and lumbar spine who were treated consecutively via a posterior surgical approach with pedicle screw fixation in which the Texas Scottish Rite Hospital system was used.

Methods. All cases were managed according to the same protocol. Follow-up review averaged 29.6 months. Radiographic evidence of osseous union and the patient's current status were analyzed. Four screws were malpositioned, and there were two dural lacerations of a nerve root and one pedicle fracture. Deep wound infections developed in five patients (5.2\%) and three patients had postoperative radicular pain. In one case, the rods disengaged from the screws; in four cases, hardware was removed but there were no broken screws. Neurological deficits improved in $85 \%$ of the patients and no patient was worse neurologically after surgery. The rate of osseous union was $96.8 \%$. Three patients developed pseudarthrosis, one of whom was asymptomatic. Back pain improved in 80 patients (85\%). A solid bone fusion, however, was not necessarily associated with decreased back pain.

Conclusions. These results support the use of pedicle screw fixation as an effective and safe procedure for fusion of the thoracolumbar and lumbar spine and support the finding that complications can be minimal when a meticulous surgical technique is used. The proper selection of patients for surgery is probably the most important factor associated with good outcomes.

Key Words * back pain * burst fracture * failed back surgery * lumbar fusion * sciatica * spinal instability * spinal instrumentation * spondylolisthesis

The usefulness of pedicle screw instrumentation has been well documented in the literature since the mid-1980s by the reports of Cotrel and colleagues,[15] Roy-Camille, et al.,[49] and Steffe, et al.[55] Its use in Europe and Latin America is well established and has been associated with a minimum potential for litigation. In the United States concerns about pedicle screw fixation of the lumbar spine reached alarmist proportions after a nonscientific and sensationalist television report questioned the indications, efficacy, and safety of this type of spinal instrumentation. Issues related to Federal Drug Administration 
(FDA) approval and the cascade of litigation that followed fueled the controversy even further.[25,30,44,51,56,58]

Nonetheless, an increasing number of spine surgeons are using this type of spinal fixation to treat a wide variety of conditions affecting the lumbar spine. Many articles have shown the superiority of pedicle screw fixation over other systems based on biomechanical properties,[2,13,17,20,29,31,34,36,39,43,46,59,61] involvement of fewer motion segments,[31,50] high rate of osseous union, $[3,20,47,51,52,55,56]$ and versatility.[3,14,20,24,51,52,56,57,60] Other reports have stressed the importance of accurate knowledge of the vertebral anatomy and the relationships between the pedicle and surrounding neural structures, indicating the risks associated with this type of instrumentation.[5,12,20,21,23,33,40,42]

In the hands of adequately trained neurosurgeons and orthopedic surgeons, this type of spinal instrumentation is safe, efficacious, and associated with better fusion rates than bone grafting without instrumentation when used for degenerative, neoplastic, or traumatic spinal instability.[2-4,17,20,47,51,52,55,56] Pedicle screw instrumentation also permits early mobilization of the patient and obviates the prolonged use of a body cast, decreasing the medical complications associated with prolonged immobilization.[4,11,20,37,49,55]

Although the biomechanical characteristics of pedicle screw fixation are excellent, the technique is associated with significant surgical risks.[6,11,20,22,57,60,62] Currently, there are no precise and clearly defined indications for the use of pedicle screw fixation in the spine.[16,20,51,56,57] Most important, information on the appropriate selection of patients who will really benefit from this type of spinal instrumentation is minimal. The FDA has not resolved the issue regarding the indications for pedicle screw fixation[25,44,56,58] and the only FDA-approved use is for L5-S1 Grade III spondylolisthesis.[23-25,56] All other uses, in spite of extensive supporting literature,[3,4,7,8,11,20,24,37,44,47,52,54,56,57,60,61] are still considered "off label."[25,51]

In an effort to clarify these issues, we reviewed the indications, complication rates, and patient outcomes accompanying pedicle screw instrumentation in the management of 95 patients who underwent pedicle screw fixation for lumbar spine instability.

\section{CLINICAL MATERIAL AND METHODS}

\section{Patient Population}

Between November 1991 and January 1997, 95 patients requiring a posterior approach for decompression and stabilization of the thoracolumbar spine were treated consecutively by the senior author (V.K.H.S.) with the Texas Scottish Rite Hospital (TSRH) pedicle screw fixation system. There were 55 women and 40 men (mean age 56.5 years; range 21-85 years). All medical records were reviewed to determine the patients' age, gender, medical history, signs, symptoms, and indications for surgery. The type of surgery, number of levels fused, and the type of hardware and grafts used intraoperatively were recorded. All radiographic studies were reviewed by the authors to determine preoperative diagnosis, possible postoperative complications, and the presence of an osseous union. Intraoperative and postoperative complications, postoperative course, and outcomes were analyzed.

All patients presented with incapacitating moderate-to-severe lower-back pain. Fifteen patients (15.8\%) exhibited no neurological signs but had evidence of spinal instability. Forty patients had unilateral radiculopathy, 27 had bilateral radiculopathy, 10 patients had cauda equina syndrome, and three patients 
were paraplegic at the time of surgery. Fifty-one patients had undergone one to three previous surgeries of the involved segment (total number of prior surgeries 67; mean number of prior surgeries 1.33). The previous surgeries consisted of 42 laminectomies, 11 discectomies, 11 fusion onlays without hardware, and three fusions with hardware.

There were 26 cases $(27.4 \%)$ of postsurgical instability, 25 cases $(26.3 \%)$ of isthmic spondylolisthesis with instability, 19 cases (20\%) of instability associated with degenerative changes and previous operations, 11 cases $(11.6 \%)$ of degenerative instability with spinal stenosis or scoliosis, six cases $(6.3 \%)$ of pseudarthrosis, five cases $(5.3 \%)$ of burst fractures in the lumbar spine, and three cases $(3.2 \%)$ of fracture-dislocation.

Follow-up visits documented in the outpatient files were reviewed, and follow-up phone interviews by the authors verified each patient's current status. The phone interviews included questions about residual back pain and/or sciatica, the persistence of paresthesia or dysesthesia, and the presence of residual weakness. Patients were questioned about their use of analgesics, muscle relaxants, and/or any other medications; follow-up evaluation by other physicians; work status; and activities of daily living. Patients were also asked to rate themselves on how well they were doing compared with before surgery (better, worse, or the same). At the end of the interview, patients were asked if they would still be willing to consent to pedicle screw instrumentation based on their current knowledge about the surgery.

\section{Management Protocol}

Preoperatively, all patients underwent complete imaging studies, including different combinations of plain radiography, computerized tomography $(\mathrm{CT})$ scanning, myelography and postmyelography CT scanning, and magnetic resonance (MR) imaging. In patients with chronic symptoms, flexion-extension films of the lumbosacral spine also were obtained.

Spinal instability was determined by using radiographic and clinical criteria. For patients with chronic spinal disease, movement on dynamic flexion-extension studies measuring $3 \mathrm{~mm}$ or greater was considered overt evidence of instability. Patients in whom no movement was demonstrated on plain films but in whom there was evidence of covert instability, such as degenerative changes on MR imaging, were also deemed to have unstable spines if their symptoms improved with external bracing. These patients were considered to have symptomatic micromotion.

For patients with chronic symptoms related to degenerative disease and with radiographic evidence of instability, conservative management was attempted before surgery; this included physical therapy, analgesic medication, muscle relaxants, bracing with a thoracolumbosacral orthosis (TLSO), and/or epidural steroid injections.

Indications for surgery for nonacute trauma included radiographic evidence of spinal instability, improvement with the use of a TLSO brace and recurrence of symptoms after use of the TLSO brace was discontinued, neurological deterioration, lack of improvement in symptoms with conservative therapy, and worsening condition despite conservative therapy.

In patients with acute spinal trauma, the Denis three-column spinal model[19] was used to determine instability. All patients with radiographic evidence of a two-column injury were considered to have unstable spines, and their decompressive surgery was based on the radiographic evidence of the compressive forces. For the most severe injuries, which affected all three columns with evidence of severe spinal instability, anterior decompression and bone grafting were initially performed and followed 
by posterior fixation.

All patients with traumatic complete spinal cord injuries were first stabilized medically and underwent elective surgery within 24 to 48 hours. Only patients who were deteriorating neurologically underwent surgery immediately after trauma. In cases in which both anterior and posterior procedures were necessary, the anterior procedure was performed first; this was followed by the posterior approach, usually within 48 hours.

\section{Surgical Technique}

For pedicle screw fixation all patients were placed prone on a radiolucent operating room table. Fluoroscopy with a C-arm image intensifier was used in all cases to permit anteroposterior and lateral views of the spine. All patients underwent somatosensory evoked potential monitor transoperatively. A course of prophylactic antibiotics was given in all patients transoperatively and for 48 hours postoperatively. With the exception of patients with neoplasms or infections, a cell-saver unit was used to reduce the need for postoperative blood transfusions.

A standard posterior approach was used in all cases. Decompressive procedures were performed if necessary, including laminectomies, foraminotomies or foraminectomies, and/or partial medial facetectomies. When necessary, discectomies were also performed as indicated by the preexisting disease and findings on the neuroimaging studies. All decompressive procedures were performed using microsurgical techniques. After adequate decompression of the spinal canal and neural elements, all segments to be fused were exposed by dissections lateral to the facets to expose the transverse processes.

The position of the pedicle was estimated using anatomical landmarks (facets and transverse processes) previously reported in the literature, $[12,15,20,21,33,49,55]$ and the estimated entry point into the pedicle and the trajectory of the screw were confirmed by fluoroscopy. Frameless stereotactic localization[32] was used as an adjunct in a few cases. Its usefulness is being evaluated.

The dorsal aspect of the pedicle was decorticated using a drill (Midas Rex, Fort Worth, TX) with an AM-8 drill bit and then manually perforated using a Steinmann pin or manual guide, with the aid of direct fluoroscopic vision in the lateral plane. The holes were enlarged using a bone awl. The appropriate tapping was performed and the pedicle screws were inserted by constant direct visualization of the medial wall of the pedicle if a laminectomy was performed. Lateral fluoroscopy was used to monitor the depth of the screw placement. Anteroposterior fluoroscopy was reserved for an intact lamina and severe scoliosis.

The screws were considered to be placed adequately when $70 \%$ of the vertebral body had been penetrated.[14] The diameter of the screws that were used was $6.5 \mathrm{~mm}$ for the lumbar region and $7 \mathrm{~mm}$ for the S-1 pedicles. If the surgeon was concerned about placement of the screws, a nerve stimulator was placed over the screw to assess the presence of motor responses.[10,20,26] In no instance was a motor response noted. After all screws were placed, anteroposterior and lateral fluoroscopy was performed to confirm the final position of the screws.

The length of the rods was estimated using a malleable endotracheal stylet, and the rods were cut and bent to fit the normal curvature of the lumbar spine. The rods were then secured to the screws with eyebolts and to each other with crosslinks to enhance rotational stability. $[4,36,43,46,56]$ The transverse processes, other dorsal bone surfaces, and facet joints were decorticated with the drill. The bone graft, which had been obtained from the iliac crest, was combined with all bone obtained during the 
decompressive procedure and placed over the decorticated surfaces. The wound was closed meticulously in multiple layers, leaving a Hemovac drain in the deep muscular plane.

Traumatic spinal deformities were always reduced using direct vision and fluoroscopic control. For lesions located at the thoracolumbar level and for patients of small stature with narrow pedicles in the high lumbar region, a construct combining laminar hooks and pedicle claws was placed, and distraction, compression, or rotational forces were applied as indicated.[4,13,31,43,56] Usually within 24 hours of surgery, CT scanning and anteroposterior and lateral radiographs of the involved spine were obtained to assess the degree of decompression and the proper placement of hardware, respectively.[12,23]

\section{Follow-Up Evaluation}

All patients were mobilized as soon as possible after surgery. If indicated, a physical therapy and rehabilitation program was initiated as soon as the patient could be allowed out of bed. All patients wore a TLSO brace 3 to 6 months after surgery. The use of the brace was discontinued after radiological confirmation of bone fusion on follow-up radiography.

Follow-up review included obtaining flexion-extension radiographs 3, 6, and 12 months after surgery. The authors reviewed all postoperative radiographs. No reports of outside studies were used to assess fusions. A fusion was considered solid when bridging of bone with trabeculations could be visualized between the transverse processes[3,6,35] with no movement on flexion-extension views. In cases in which fusion was questionable, CT scans and/or tomograms were obtained to evaluate the fusion.[35] The mean duration of follow up was 29.6 months (range 6-67 months).

\section{RESULTS}

\section{Surgical Outcomes}

At the time of final follow-up evaluation, one patient had died and five patients could not be located. Eighty-nine patients (94\%) were available for the complete evaluation, including the 3-, 6-, and 12-month follow-up examinations.

As indicated by their myelography CT or MR imaging studies, 86 patients underwent a posterior decompressive procedure, laminectomy, foraminotomy, or discectomy. Two trauma patients underwent transpedicular decompression of a burst fracture that encroached on the spinal canal. Five trauma patients required an anterior decompressive procedure before the posterior fusion: four underwent anterior internal fixation and one required only a graft. Reduction of a vertebral abnormality was attempted in six trauma patients. Reduction failed in one patient with a fracture-dislocation at L3-4, and an anterior procedure was performed to realign the spine adequately. No reduction was attempted in patients with spondylolisthesis; their spines were fused in situ.

The number of levels involved was as follows: two levels were fused in 48 cases, three levels in 29 cases, four levels in eight cases, five levels in five cases, and six levels in two cases. Seven, eight, and 10 levels were fused in one case each. The mean number of levels fused was 2.9. The most frequently fused level was L4-5 (25 cases). In 12 cases L4-S1 was fused. Instrumentation was placed in the lower thoracic spine in five cases and in the remaining cases in the lumbar spine (61 cases) and the lumbosacral spine (29 cases).

Overall, 434 intrapedicular screws were placed (mean 4.5 per patient). Eight pedicle hooks, two 
transverse hooks, and 10 laminar hooks were applied. In one case sublaminar wires were used.

Only two patients received allograft for posterolateral fusion. One patient experienced a previous infection in the disc space and the graft was considered unnecessary. In the remaining 92 patients, iliac bone autograft was used for posterolateral fusion.

There were no operative deaths. One patient died 13 months after surgery of unrelated causes.

\section{Complications of Treatment}

Intraoperative. Four screws were malpositioned ( $0.9 \%$ of the screws) but this was recognized immediately and the screws were repositioned during the same procedure. In two cases, the dura of the nerve root was lacerated ( $0.5 \%$ of the screws; $2.1 \%$ of the patients), but it was repaired immediately during the same surgery. In one case, a screw fractured a pedicle. The screw was removed and another was placed instead at an upper level (as was the contralateral screw). One patient with a coagulation disorder bled excessively and required multiple transfusions.

Postoperative. Five patients (5.3\%) developed deep wound infections. The infections were managed with surgical debridement, a closed irrigation system, and intravenous administration of antibiotic medication. However, these measures failed to control infection in two patients and, therefore, their hardware was removed. Both patients had developed solid bone fusion by that time. One of these patients developed septic shock and required a prolonged stay in the intensive care unit; however, eventually that patient achieved a good recovery. At long-term follow-up review, all five patients who experienced infection have done well.

Five patients (5.3\%) experienced a subcutaneous fluid collection that improved spontaneously. One patient had a subcutaneous hematoma that required surgical drainage. Three patients $(3.2 \%)$ developed radicular pain not noted before surgery, but they had no motor deficits. Two patients improved. In the patient with continued pain, an electromyographic study was negative for radiculopathy and MR imaging showed no compression of the involved root.

Medical complications included a urinary infection in three patients, deep thrombophlebitis in one patient, postoperative ileus in one patient, and postoperative delirium in one patient. All of these patients improved after adequate management.

Delayed. There were several late complications related to the hardware. In one patient, the assembly disengaged after 8 months, but no symptoms or signs developed and plain radiography showed a solid osseous union. Hardware was removed in four patients (4.2\%): two for infection, as discussed earlier, and the other two for lower-back pain. The latter two patients improved dramatically after the hardware was removed. No broken screws were noted.

\section{Rate of Pseudarthrosis}

The overall fusion rate was $96.8 \%$. Three patients (3.2\%), all of whom had undergone previous surgeries on the involved segment, developed pseudarthrosis. Two of these patients $(2.1 \%)$ were symptomatic. One, a 60-year-old woman with congenital dwarfism and three previous surgeries for spinal stenosis, underwent a L3-4 fusion. She developed back pain 8 months after the fusion and her radiographic studies showed pseudarthrosis. After undergoing an anterior procedure, which included a corpectomy and placement of a Harm's cage and a Z-plate, she improved. The other patient, a man with a history of 
smoking 40 cigarettes per day and a previous back surgery, underwent a pedicular L4-5 fusion with autograft. He developed pseudarthrosis 1 year after surgery with recurrence of his back pain but declined further intervention. One patient still has not developed a solid osseous union on plain radiographs 2 years after surgery, but he remains asymptomatic. No adjacent segmental instability was noted postoperatively in our patients; thus, no patient required reoperation to extend the fusion.

\section{Neurological Deficits}

Of the 67 patients with unilateral or bilateral radiculopathy, 58 (86.6\%) completely recovered their motor or sensory function. The remaining nine patients showed little or no improvement but none worsened after surgery. Of the 10 patients with cauda equina syndrome, four patients recovered completely and six patients improved but still had some neurological deficits. All 10 patients can now walk independently or with a cane. The three patients who had no motor function before surgery did not improve.

\section{Back Pain: Functionality}

Despite the $96.8 \%$ fusion rate, back pain resolved completely in only $44.6 \%$ of the 94 surviving patients (Table 1). Back pain failed to resolve in $15.2 \%$ of the 92 patients in whom solid fusions were formed. Overall, 14 patients experienced no improvement, seven of whom stated that their pain was worse after surgery. The remaining $80(85 \%)$ of the 94 survivors improved substantially $(40.4 \%)$ [61] or completely $(44.7 \%),[32]$ and all returned to work or were independent and satisfied with their surgical outcome. Postoperatively, all patients who experienced no pain or some pain but had returned to work or were independent $(85 \%)$ had discontinued their use of all narcotic pain medication. Of all patients, $11(11.6 \%)$ said they would not have surgery if faced with the same decision again and $88.4 \%$ of the patients said they would still choose surgery knowing what they now knew regarding their postoperative course and recovery.

\begin{tabular}{|cc|}
\hline \multicolumn{2}{|c|}{$\begin{array}{c}\text { TABLE 1 } \\
\text { CURRENT STATUS OF 94 SURVING PATIENTS' BACK PAIN AF TER PED KLE } \\
\text { SCRREW FIXATION }\end{array}$} \\
\hline \hline \multicolumn{1}{|c|}{ Outcome } & No. of Patierts (96) \\
\hline no pain & $42(44.7)$ \\
some pain but returned to work or independent & $38(40.4)$ \\
same pain as before surgery & $7(7.4)$ \\
morsened pain & $7(7.4)$ \\
\hline
\end{tabular}

\section{DISCUSSION}

\section{Regulatory Issues}

Pedicle screw fixation has been in clinical use in Europe for more than 20 years, and the efficacy and safety of these devices have been amply documented.[3,14,15,28,49,53,54] Although technical considerations and strategies for minimizing complications with pedicle screw systems are well known, $[3,5,7,15,20,23,28,42,56,58,62]$ the widespread use of pedicle screw fixation in the United States is still in an "off label" category.[25,51] In 1994, a special panel recommended that the FDA expand the indications for pedicle screws to include degenerative disorders and trauma.[24,44,57] The FDA is currently evaluating upgrade of these devices from Class III to Class II. Pedicle screws are approved by the FDA for use in Grade III spondylolisthesis at L5-S1.[23-25,56] Despite these restrictions and medicolegal concerns,[25,51,56] many spinal surgeons use pedicle screw systems because of the benefits 
they provide.[3,20,47,52,54,58]

\section{Patient Outcomes}

This study further supports the use of pedicle screw instrumentation for traumatic and degenerative spinal instability. In this review, screws that were malpositioned $(<1 \%)$ were always detected intraoperatively. The risk of malpositioning can be almost completely eliminated with careful preoperative planning, [5] accurate knowledge of the spinal anatomy,[23,40] and the use of the image intensifier during all the steps of screw placement.[20] At our institution, visual inspection of the medial wall of the pedicle is mandatory. Stimulating a screw with a nerve stimulator can also confirm contact between it and a nerve.[10,20,26] Some authors have reported good results after using only lateral films intraoperatively, but their rates of malpositioning the screws are higher than those of other series.[23,45] In our series, fluoroscopy helped avoid malpositioning of screws as evidenced by postoperative radiography and CT scanning. The two dural sleeve injuries occurred early in the series.

Infection can be life threatening and must be managed carefully.[22,27] Our postoperative infection rate (5.2\%) was comparable to rates reported in other series.[3,7,11,18,20,22,27,37,52,54-56,58,60] Infection caused the hardware to be removed in two patients despite aggressive treatment. In no case, however, did it cause pseudarthrosis or failure of fusion.

Clinical factors that contribute to a higher infection rate include the number of personnel in the operating room, the use of an image intensifier or other localizing and monitoring devices, prolonged surgical times, previous surgery, presence of diabetes mellitus or morbid obesity, and the formation and accumulation of postoperative hematoma or serum.[22,27,45] Meticulous aseptic technique and decreasing the duration of surgery probably can diminish infection rates.[27]

All three patients who experienced postoperative radicular pain had undergone previous operations. Consequently, the dissections that preceded screw placement were difficult because scar tissue obscured the normal anatomy. These patients' pain was therefore likely due to nerve root manipulation during the decompressive part of the procedure and not necessarily related to screw placement. Furthermore, on postoperative neuroimaging studies we could see that the screws were positioned correctly. Eventually, two of these three patients recovered completely.

The medical complications encountered were similar to those expected and associated with major surgery. None of the medical complications interfered with the final outcome.

The low rate of hardware breakage in this series, also comparable to those of other series, $[3,9,20,22,41,52,56,57,60]$ partially reflects the use of large diameter screws and patients' postoperative use of a TLSO brace. Not all surgeons require their patients to wear braces after pedicle screw instrumentation.[54,56] External immobilization, however, probably immobilizes the spine to some degree and prevents patients from being involved in excessive physical activities while they recuperate.

Bone scans in the four patients in whom hardware wasremoved showed increased uptake of isotope near the screws. These findings suggest loosening and "micromotion" of the screws.[35] Some authors have proposed micromotion as a mechanism to explain persistent lower-back pain after fusion. Factors such as bursitis or inflammatory changes surrounding the screws have also been postulated.[51,52]

The $96.8 \%$ fusion rate in this series compares favorably with rates in other 
series.[3,7,8,11,18,20,47,49,52,54,56,57,60,61] Routine plain and flexion-extension radiographs are needed to determine the presence of pseudarthrosis, although these studies may not always correlate with the presence of pseudarthrosis.[6,35,47,51] The sensitivity of plain films may only be 35 to 67\%.[6,35] Risk factors for pseudarthrosis include cigarette smoking, osteoporosis, obesity, previous fusion failure, and the use of allograft.[1,6,34,39,47,51,52] Some authors have advocated the use of an implanted bone-growth stimulator[48] and anterior fusion or posterior interbody fusion combined with pedicle screw fusion to enhance the possibility of obtaining a solid bone fusion in patients at high risk for pseudarthrosis. $[8,18,22]$

As in other published series, the resolution of back pain does not necessarily relate to the rate of fusion.[3,6,20,44,49,52,56,61] The reasons are unclear but could be related to a combination of factors: persistent arthritis, pseudarthrosis, inadequate decompression, insufficient number of levels fused, psychological factors, secondary gain, and problems unrelated to the spine.[35,38]

The resolution of sciatica and the good neurological outcome achieved emphasize the importance of extensive intraoperative decompression that correlates with the regions of compression visualized on the preoperative imaging studies.[38,47] Spinal realignment and prevention of further deformity in trauma patients play an important role in the adequate rehabilitation of the injured neural structures.[4,11,42,53] Patients with instability and extensive destruction of the anterior column and those with anterior compression of the spinal canal benefit from anterior decompression and bone grafting with or without anterior instrumentation followed by posterior pedicle screw instrumentation.[11,17,41,43,50] Application of pedicle screws in the thoracic spine is associated with a higher risk of neurological injury when the pedicles are thin. $[15,16,28,33,40]$ In such cases, laminar, transverse process, or pedicle hooks are used and attached to the pedicle screws in the lumbosacral spine.[4,13,28,31,43,46,56]

Patients with spondylolisthesis were treated with decompression and fusion in situ. $[8,47]$ Reduction of the spondylolisthesis was not necessary, as other authors have also reported.[8,20]

\section{CONCLUSIONS}

This study supports the usefulness of pedicle screw instrumentation. The procedure was associated with a low morbidity rate and no death. The fusion rate was high (96.8\%) and improvement was good. Pedicle screw fixation is indicated for the management of instability of the lower thoracic, lumbar, and lumbosacral spine. The appropriate selection of patients for surgery and the proper identification of patients most likely to improve after fusion are probably the most difficult and important issues to resolve. Identification of risk factors or patients' characteristics that predispose to pseudarthrosis is also important. Identification of patients most likely to improve and risk factors for pseudarthrosis should be the subject of more extensive research. In our series, 84 patients (almost 90\%) stated that if faced again with making a decision concerning surgery, but knowing what recovery was like, they would still opt to have the instrumentation placed.

\section{References}

1. An HS, Lynch K, Toth J: Prospective comparison of autograft vs. allograft for adult posterolateral lumbar spine fusion: differences among freeze-dried, frozen, and mixed grafts. J Spinal Disord 8:131-135, 1995

2. Asazuma T, Stokes IAF, Moreland MS, et al: Intersegmental spinal flexibility with lumbosacral 
instrumentation. An in vitro biomechanical investigation. Spine 15:1153-1158, 1990

3. Bailey SI, Bartolozzi P, Bertagnoli R, et al: The BWM spinal fixator system. A preliminary report of a 2-year prospective, international multicenter study in a range of indications requiring surgical intervention for bone grafting and pedicle screw fixation. Spine 21:2006-2015, 1996

4. Benzel EC, Kesterson L, Marchand EP: Texas Scottish Rite Hospital rod instrumentation for thoracic and lumbar spine trauma. J Neurosurg 75:382-387, 1991

5. Bernard TN Jr, Seibert CE: Pedicle diameter determined by computed tomography. Its relevance to pedicle screw fixation in the lumbar spine. Spine 17 (Suppl 6):160-163, 1992

6. Blumenthal SL, Gill K: Can lumbar spine radiographs accurately determine fusion in postoperative patients? Correlation of routine radiographs with a second surgical look at lumbar fusions. Spine 18:1186-1189, 1993

7. Blumenthal S, Gill K: Complications of the Wiltse Pedicle Screw Fixation System. Spine 18:1867-1871, 1993

8. Boos N, Marchesi D, Zuber K, et al: Treatment of severe spondylolisthesis by reduction and pedicular fixation. A 4-6-year follow-up study. Spine 18:1655-1661, 1993

9. Brantley AGU, Mayfield JK, Koeneman JB, et al: The effects of pedicle screw fit. An in vitro study. Spine 19:1752-1758, 1994

10. Calancie B, Lebwohl N, Madsen P, et al: Intraoperative evoked EMG monitoring in an animal model. A new technique for evaluating pedicle screw placement. Spine 17 (Suppl 8):1229-1235, 1992

11. Carl AL, Tromanhauser SG, Roger DJ: Pedicle screw instrumentation for thoracolumbar burst fractures and fracture-dislocations. Spine 17:317-324, 1992

12. Castro WHM, Halm H, Jerosch J, et al: Accuracy of pedicle screw placement in lumbar vertebrae. Spine 21:1320-1324, 1996

13. Chiba M, McLain RF, Yerby SA, et al: Short-segment pedicle instrumentation. Biomechanical analysis of supplemental hook fixation. Spine 21:288-294, 1996

14. Cigliano A, de Falco R, Scarano E, et al: A new instrumentation system for the reduction and posterior stabilization of unstable thoracolumbar fractures. Neurosurgery 30:208-217, 1992

15. Cotrel Y, Dubousset J, Guillaumat M: New universal instrumentation in spinal surgery. Clin Orthop 227:10-23, 1988

16. Crawford MJ, Esses SI: Indications for pedicle fixation. Results of NASS/SRS faculty questionnaire. North American Spine Society and Scoliosis Research Society. Spine 19:2584-2589, 1994

17. Cunningham BW, Sefter JC, Shono Y, et al: Static and cyclical biomechanical analysis of pedicle screw spinal constructs. Spine 18:1677-1688, 1993

18. Davne SH, Myers DL: Complications of lumbar spinal fusion with transpedicular instrumentation. Spine 17 (Suppl 6): 184-189, 1992 
19. Denis F: Spinal instability as defined by the three-column spine concept in acute spinal trauma. Clin Orthop 189:65-76, 1984

20. Dickman CA, Fessler RG, MacMillan M, et al: Transpedicular screw-rod fixation of the lumbar spine: operative technique and outcome in 104 cases. J Neurosurg 77:860-870, 1992

21. Ebraheim NA, Rollins JR Jr, Xu R, et al: Projection of the lumbar pedicle and its morphometric analysis. Spine 21:1296-1300, 1996

22. Esses SI, Sachs BL, Dreyzin V: Complications associated with the technique of pedicle screw fixation. A selected survey of ABS members. Spine 18:2231-2239, 1993

23. Farber GL, Place HM, Mazur RA, et al: Accuracy of pedicle screw placement in lumbar fusions by plain radiographs and computed tomography. Spine 20:1494-1499, 1995

24. Garfin SR: Historical cohort study of pedicle screw fixation in thoracic, lumbar, and sacral spine fusions. Spine 19 (Suppl 20):2254S-2255S, 1994 (Editorial)

25. Garfin SR, Yuan HA: Food and Drug Administration regulation of spinal implant fixation devices. Clin Orthop 335:32-38, 1997

26. Glassman SD, Dimar JR, Puno RM, et al: A prospective analysis of intraoperative electromyographic monitoring of pedicle screw placement with computed tomographic scan confirmation. Spine 20:1375-1379, 1995

27. Glassman SD, Dimar JR, Puno RM, et al: Salvage of instrumented lumbar fusions complicated by surgical wound infection. Spine 21:2163-2169, 1996

28. Graziano GP: Cotrel-Dubousset hook and screw combination for spine fractures. J Spinal Disord 6:380-385, 1993

29. Gurr KR, McAfee PC, Shih CM: Biomechanical analysis of posterior instrumentation systems after decompressive laminectomy. An unstable calf-spine model. J Bone Joint Surg (Am) 70:680-691, 1988

30. Heim SE, Vinkler JA: Recent medical-legal proceedings and their effect on the practice of medicine in Chicago. J Spinal Disord 8:419-421, 1995

31. Johnston CE II, Welch RD, Baker KJ, et al: Effect of spinal construct stiffness on short segment fusion mass incorporation. Spine 20:2400-2407, 1995

32. Kalfas IH, Kormos DW, Murphy MA, et al: Application of frameless stereotaxy to pedicle screw fixation of the spine. J Neurosurg 83:641-647, 1995

33. Kothe R, O'Holleran JD, Liu W, et al: Internal architecture of the thoracic pedicle. An anatomic study. Spine 21:264-270, 1996

34. Kumano K, Hirabayashi S, Ogawa Y, et al: Pedicle screws and bone mineral density. Spine 19:1157-1161, 1994

35. Larsen JM, Rimoldi RL, Capen DA, et al: Assessment of pseudarthrosis in pedicle screw fusion: a prospective study comparing plain radiographs, flexion/extension radiographs, CT scanning, and bone scintigraphy with operative findings. J Spinal Disord 9:117-120, 1996 
36. Lim TH, Eck JC, An HS, et al: Biomechanics of transfixation in pedicle screw instrumentation. Spine 21:2224-2229, 1996

37. Mac Millan MM, Cooper R, Haid R: Lumbar and lumbosacral fusions using Cotrel-Dubousset pedicle screws and rods. Spine 19:430-434, 1994

38. Markwalder TM, Saager C, Reulen HJ: "Isthmic" spondylolisthesis--an analysis of the clinical and radiological presentation in relation to intraoperative findings and surgical results in 72 consecutive cases. Acta Neurochir 110:154-159, 1991

39. McAfee PC, Farey ID, Sutterlin CE, et al: 1989 Volvo Award in basic science. Device-related osteoporosis with spinal instrumentation. Spine 14:919-926, 1989

40. McCormack BM, Benzel EC, Adams MS, et al: Anatomy of the thoracic pedicle. Neurosurgery 37:303-308, 1995

41. McLain RF, Sparling E, Benson DR: Early failure of short-segment pedicle instrumentation for thoracolumbar fractures. A preliminary report. J Bone Joint Surg (Am) 75:162-167, 1993

42. Misenhimer GR, Peek RD, Wiltse LL, et al: Anatomic analysis of pedicle cortical and cancellous diameter as related to screw size. Spine 14:367-372, 1989

43. Moreland DB, Egnatchik JG, Bennett GJ: Cotrell-Dubousset instrumentation for the treatment of thoracolumbar fractures. Neurosurgery 27:69-73, 1990

44. Mulholland RC: Pedicle screw fixation in the spine. J Bone Joint Surg (Br) 76:517-519, 1994 (Editorial)

45. Odgers CJ IV, Vaccaro AR, Pollack ME, et al: Accuracy of pedicle screw placement with the assistance of lateral plain radiography. J Spinal Disord 9:334-338, 1996

46. Pintar FA, Maiman DJ, Yoganandan N, et al: Rotational stability of a spinal pedicle screw/rod system. J Spinal Disord 8:49-55, 1995

47. Ricciardi JE, Pflueger PC, Isaza JE, et al: Transpedicular fixation for the treatment of isthmic spondylolisthesis in adults. Spine 20:1917-1922, 1995

48. Rogozinski A, Rogozinski C: Efficacy of implanted bone growth stimulation in instrumented lumbosacral spinal fusion. Spine 21:2479-2483, 1996

49. Roy-Camille R, Saillant G, Mazel C: Internal fixation of the lumbar spine with pedicle screw plating. Clin Orthop 203:7-17, 1986

50. Sasso RC, Cotler HB: Posterior instrumentation and fusion for unstable fractures and fracture-dislocations of the thoracic and lumbar spine. A comparative study of three fixation devices in 70 patients. Spine 18:450-460, 1993

51. Schnee CL, Freese A, Ansell LV: Outcome analysis for adults with spondylolisthesis treated with posterolateral fusion and transpedicular screw fixation. J Neurosurg 86:56-63, 1997

52. Schwab FJ, Nazarian DG, Mahmud F, et al: Effects of spinal instrumentation on fusion of the lumbosacral spine. Spine 20:2023-2028, 1995 
53. Sjostrom L, Karlstrom G, Pech P, et al: Indirect spinal canal decompression in burst fractures treated with pedicle screw instrumentation. Spine 21:113-123, 1996

54. Stambough JL: Cotrel-Dubousset instrumentation and thoracolumbar spine trauma: a review of 55 cases. J Spinal Disord 7:461-469, 1994

55. Steffee AD, Biscup RS, Sitkowski DJ: Segmental spine plates with pedicle screw fixation. A new internal fixation device for disorders of the lumbar and thoracolumbar spine. Clin Orthop 203:45-53, 1986

56. Vaccaro AR, Garfin SR: Internal fixation (pedicle screw fixation) for fusions of the lumbar spine. Spine 20 (Suppl 24):157S-165S, 1995

57. Yahiro MA: Comprehensive literature review. Pedicle screw fixation devices. Spine 19 (Suppl 20):2274S-2278S, 1994

58. Yahiro MA: Review of the "Historical Cohort Study of Pedicle Screw Fixation in Thoracic, Lumbar, and Sacral Spinal Fusions" report. Spine 19 (Suppl 20):2297-2299, 1994

59. Yamagata M, Kitahara H, Minami S, et al: Mechanical stability of the pedicle screw fixation systems for the lumbar spine. Spine 17 (Suppl 3):51-54, 1992

60. Yuan HA, Garfin SR, Dickman CA, et al: A historical cohort study of pedicle screw fixation in thoracic, lumbar, and sacral spinal fusions. Spine 19 (Suppl 20):2279S-2296S, 1994

61. Zdeblick TA: A prospective, randomized study of lumbar fusion. Preliminary results. Spine 18:983-991, 1993

62. Zindrick MR: The role of transpedicular fixation systems for stabilization of the lumbar spine. Orthop Clin North Am 22:333-344, 1991

Manuscript received September 3, 1997.

Accepted in final form May 4, 1998.

Current address for Dr. Gomez: Universidad de Antioquia, Medellin, Colombia.

Current address for Dr. Masferrer: Mountain Neurosurgical Associates, Colorado Springs, Colorado.

Address reprint requests to: Volker K. H. Sonntag, M.D., c/o Neuroscience Publications, Barrow Neurological Institute, 350 West Thomas Road, Phoenix, Arizona 85013. email: neuropub@mha.chw.edu.

Return to Journal of Neurosurgery Table of Contents.

Table of Contents Return to Na//OC(i) 